\title{
Infección por Hepatitis No A / No B posterior a accidente biológico en personal de enfermería. A propósito de un caso
}

\author{
No A/ No B Hepatitis infection after a biological accident in nursing staff. A \\ Report of a case
}

\author{
Finol Muñoz, Alexander '; Ortega Marín, Gloria '; Domínguez Fernández, Julián 1,2; Rivero Colina, Johanna '; \\ Querol Fernández, José ${ }^{1}$
}

1. Hospital Universitario de Ceuta. España.

2. Unidad Docente Multiprofesional de Salud Laboral. Hospital Universitario de Ceuta. España.

Recibido: 18-07-14

Aceptado: 07-08-14

\section{Correspondencia}

Alexander José Finol Muñoz.

Hospital Universitario de Ceuta

Loma Colmenar s/n. ${ }^{\circ}$

Edificio 2. Planta - 1 .

51003. Ceuta. España

Correo electrónico: finolito@hotmail.com

\section{Resumen}

Los accidentes por exposición percutánea, suponen aproximadamente un tercio de los accidentes laborales de los trabajadores de salud. Por categorías profesionales, la enfermería presenta la mayor frecuencia. Los agentes más importantes por su frecuencia y perjuicio son: el VHB, VIH y VHC. En la década de los 60 y 70 , los científicos desarrollaron análisis de sangre para identificar hepatitis B (1963) y hepatitis A (1973), pero muchas de las muestras de sangre tomadas para detectar enfermedades producidas tras las transfusiones resultaron negativas tanto para hepatitis A como la hepatitis B. Caso Clínico: Mujer, 27 años de edad, profesional de enfermería en área de Medicina Interna, sufre accidente laboral tipo pinchazo con abbocath durante jornada del día 23/11/1989. Se realiza revisión de historia del paciente fuente y las respectivas pruebas confirmatorias, sin encontrarse datos de enfermedades transmisibles. Así mismo las analíticas realizadas en el enfermo y en la trabajadora resultaron negativos los marcadores virales para Hepatitis. 15 días posteriores, presenta clínica sugestiva de hepatitis. Se realiza analítica y seriado de enzimas hepáticas, evidenciándose hipertransaminemia y serología para hepatitis A y B negativos. Se inicia tratamiento médico; con buena evolución clínica y de laboratorio es dada de alta. Posteriormente en 1996, se realiza reconocimiento médico laboral, encontrándose positividad para VHC. En 2004 por antecedentes de accidente biológico, se localiza el paciente fuente, repitiéndose el control serológico, resultando positivo para el mismo virus, pudiendo ahora ser declarado como accidente laboral y enfermedad profesional, por demostrarse la relación entre paciente fuente y la trabajadora.

Med Segur Trab (Internet) 2014; 60 (236) 600-607

Palabras clave: VHC, Enfermedad profesional, Accidente laboral, Riesgo biológico.

\section{Abstract}

Cutaneous exposure accidents, suppose approximately a third of the accidents at work of health workers. For professional categories, the nursing presents the major frequency. The most important agents for its frequency and prejudice are: the VHB, HIV and VHC. In the decade of the 60 and 70, the scientists 
developed blood test to identify hepatitis B (1963) and hepatitis A (1973), but many of the samples of blood taken to detect diseases produced after the transfusions turned out to be negative for hepatitis A and hepatitis B. Clinical case: Woman, 27 years old, professional of nursing in area of Internal Medicine, suffers accident at work type prick with abbocath during the day 23/11/1989. The medical history of source patient is reviewed and the respective confirmatory tests, without being information of contagious diseases. Likewise, the analytical realized in the patient and in the worker turned out to be negative the viral scoreboards for Hepatitis. 15 days later, the nurse presents suggestive clinic of hepatitis. It is realized analytical and checked of enzymes hepatic, being demonstrated hipertransaminemia and serologia for hepatitis A and B negatives. Medical treatment begins; with good clinical laboratory evolution. She is send home. Later in 1996, it is realized medical labor recognition, being positive for VHC. In 2004 for precedents of biological accident, source patient is located, repeating the serological test, turning out to be positive. Being able to declare now as accident at work and occupational disease, for the relation demostrated between source patient and the worker.

Med Segur Trab (Internet) 2014; 60 (236) 600-607

Keywords: VHC, Occupational disease, Work accident, Biological risk. 


\section{INTRODUCCIÓN}

La adquisición de infecciones por patógenos que circulan por la sangre a través de accidentes punzo-cortantes o exposición de mucosas o piel no intacta a fluidos infectantes, constituye uno de los principales riesgos profesionales para los trabajadores de salud.

Los accidentes por exposición percutánea, suponen aproximadamente un tercio de los accidentes laborales de estos trabajadores. Por categorías profesionales, la de enfermería presenta la mayor frecuencia ${ }^{1}$.

Los accidentes por exposición percutánea incluyen aquellos que conllevan una penetración a través de la piel por una aguja u otro objeto punzante o cortante contaminado con sangre, con otro fluido que contenga sangre visible, con otros fluidos potencialmente infecciosos o con tejidos de un paciente. Dentro de los fluidos potencialmente infecciosos se incluyen los líquidos cefalorraquídeo, sinovial, pleural, peritoneal, pericárdico y amniótico, así como el semen y las secreciones vaginales ${ }^{2}$.

Se han reportado más de 20 agentes diferentes con la capacidad de transmitirse por esta vía, dentro de los que podemos mencionar al virus de la inmunodeficiencia humana (VIH), virus de la hepatitis B (VHB), virus de la hepatitis C (VHC), virus ébola, virus del dengue, virus herpes 1 y 2, virus de la varicela, Treponema pallidum, Brucella sp, Corynebacterium, Rickettsia sp, Leptospira sp, micobacterias incluyendo tuberculosis, Mycoplasma, Babesia, Plasmodium, Toxoplasma, Cryptococcus y Blastomyces. Sin embargo, los agentes más importantes por su frecuencia y perjuicio son: el VHB, el VIH y el VHC ${ }^{1}$.

El virus de la hepatitis C afecta a más de 170 millones de personas en todo el mundo. Aunque existen variaciones en la prevalencia de anticuerpos anti-VHC, años atrás se comunicó que entre un $0,5 \%-1,5 \%$ de los donantes voluntarios de sangre eran positivos. En cuanto a la población general, la positividad para anti-VHC se cifra en torno al 2-3 \%, siendo más prevalente a partir de los 30 años. La principal vía de transmisión del VHC es parenteral por exposición a sangre infectada. La transfusión de hemoderivados y las personas que comparten jeringuillas con adicción a drogas por vía parenteral (ADVP) son los más prevalentes².

En la década de los 60 y 70, los científicos desarrollaron análisis de sangre para identificar la hepatitis B (1963) y la hepatitis A (1973), pero muchas de las muestras de sangre tomadas para detectar enfermedades producidas tras las transfusiones resultaron negativas tanto para hepatitis A como la hepatitis B. Dado que el modo de transmisión (transfusión de sangre) era el mismo, los científicos clasificaron los casos no identificados como hepatitis no A/ no B. En la actualidad, se cree que el $90-95 \%$ de los casos que entonces se clasificaron de esa forma, en realidad eran de Hepatitis $\mathrm{C}^{3}$.

\section{Caso Clínico}

Mujer, 27 años de edad, profesional de enfermería en área de hospitalización de Medicina Interna, sufre accidente laboral tipo pinchazo con abbocath durante jornada de trabajo el día 23/11/1989. Por lo que se realiza revisión de caso clínico del paciente fuente y las respectivas pruebas confirmatorias, sin encontrarse información en la historia clínica de datos de enfermedades transmisibles. Así mismo las analíticas realizadas en el enfermo y en la trabajadora resultaron negativos los marcadores virales para Hepatitis. 15 días posteriores, presenta alza térmica cuantificada en $40 .^{\circ}$, dolor abdominal ubicado en hipocondrio derecho, de leve intensidad, continuo, sin irradiación, no cede con cambios posturales ni con tratamiento ambulatorio, acompañándose de nauseas, vómitos y astenia. Niega coluria, acolia e ictericia, por lo que acude a servicio de urgencia y se decide su ingreso. Durante su estancia se realiza analítica y seriado de enzimas hepáticas, evidenciándose hipertransaminemia y serología para hepatitis A y B negativos. (Ver Tabla 1). Se inicia tratamiento médico con Konakión ${ }^{\circledR}$, Lisozima ${ }^{\circledR}$, Nervobión ${ }^{\circledR}$ y Gammaglobulina Humana Polivalente, de la misma forma fue vacunada frente a la Hepatitis A y Hepatitis B. Con buena evolución clínica y de laboratorio, es dada de alta con seguimiento médico.

Posteriormente en el año 1996, se realiza reconocimiento médico laboral, encontrándose en los resultados analíticos, positividad para VHC y negativo para VIH. En 
el año 2004 en vista de los antecedentes personales de la trabajadora de accidente biológico en el año 1989, se localiza el paciente fuente, repitiéndose el control serológico, resultando positivo para el mismo virus, pudiendo ser declarado aquel evento ocurrido hace 15 años, como accidente laboral y enfermedad profesional, por demostrarse la relación entre el paciente fuente y la trabajadora.

Tabla 1

\begin{tabular}{|c|c|c|c|c|c|c|c|c|}
\hline & $5 / 12 / 89$ & 11/12/89 & 12/12/89 & 13/12/89 & 15/12/89 & 16/12/89 & 18/12/89 & 23/01/90 \\
\hline Leucos & & 4.6 & & & & & 7.6 & 8.2 \\
\hline $\mathrm{Hg}$ & & $14.5 \mathrm{~g} / \mathrm{dl}$ & & & & & $13.7 \mathrm{~g} / \mathrm{dl}$ & $14.9 \mathrm{~g} / \mathrm{dl}$ \\
\hline$\overline{\text { HCT }}$ & & $45.7 \%$ & & & & & $39.6 \%$ & $50.8 \%$ \\
\hline VCM & & $85.0 \mathrm{fl}$. & & & & & $88.8 \mathrm{fl}$. & $88.5 \mathrm{fl}$. \\
\hline Plaq & & 318 & & & & & 243 & 279 \\
\hline Neutrof & & 66 & & & & & 36 & 54 \\
\hline Linfo & & 26 & & & & & 58 & 46 \\
\hline Glucosa & & $74 \mathrm{mg} / \mathrm{dl}$ & & & $86 \mathrm{mg} / \mathrm{dl}$ & & & 98 \\
\hline$\overline{\text { PCR }}$ & & & Negativo & & & & & \\
\hline$\overline{\text { ASLO }}$ & & & $166 \mathrm{U} / \mathrm{L}$ & & & & & \\
\hline$\overline{\mathbf{A R}}$ & & & Negativo & & & & & \\
\hline$\overline{\text { GOT }}$ & & $103 \mathrm{U} / \mathrm{L}$ & & $7350 \mathrm{U} / \mathrm{L}$ & $3920 \mathrm{U} / \mathrm{L}$ & & $350 \mathrm{U} / \mathrm{L}$ & $115 \mathrm{U} / \mathrm{L}$ \\
\hline$\overline{\text { GTP }}$ & & $188 \mathrm{U} / \mathrm{L}$ & & $6350 \mathrm{U} / \mathrm{L}$ & $6540 \mathrm{U} / \mathrm{L}$ & & $2250 \mathrm{U} / \mathrm{L}$ & $181 \mathrm{U} / \mathrm{L}$ \\
\hline \multicolumn{9}{|l|}{$\overline{\mathrm{CPK}}$} \\
\hline Gamma & & & & $279 \mathrm{U} / \mathrm{L}$ & & & & \\
\hline Fosf. Alc & & $96 \mathrm{U} / \mathrm{L}$ & & $234 \mathrm{U} / \mathrm{L}$ & & & & $170 \mathrm{U} / \mathrm{L}$ \\
\hline Amilasa & & $106 \mathrm{U} / \mathrm{L}$ & & $166 \mathrm{U} / \mathrm{L}$ & & & & \\
\hline Bili TOTAL & & $0.28 \mathrm{mg} / \mathrm{dl}$ & & $1.40 \mathrm{mg} / \mathrm{dl}$ & $3.4 \mathrm{mg} / \mathrm{dl}$ & & & $0.91 \mathrm{mg} / \mathrm{dl}$ \\
\hline Bili DIREC & & & & $0.60 \mathrm{mg} / \mathrm{dl}$ & $2.92 \mathrm{mg} / \mathrm{dl}$ & & & $0.7 \mathrm{mg} / \mathrm{dl}$ \\
\hline$\overline{\text { TPT }}$ & & & & & $36.7 \mathrm{seg}$ & & $86.3 \%$ & 29 seg \\
\hline$\overline{\text { TPP }}$ & & & & $33.5 \%$ & $45.7 \%$ & & $24.5 \mathrm{seg}$ & $86 \%$ \\
\hline Urea & & $23 \mathrm{mg} / \mathrm{dl}$ & & & $27 \mathrm{mg} / \mathrm{dl}$ & & & $19 \mathrm{mg} / \mathrm{dl}$ \\
\hline Creati. & & $0.89 \mathrm{mg} / \mathrm{dl}$ & & & & & & \\
\hline $\mathrm{Na}+$ & & $141 \mathrm{mEq} / 1$ & & & & & & $142 \mathrm{mEq} / \mathrm{l}$ \\
\hline$\overline{\mathbf{K}}+$ & & $4.4 \mathrm{mEq} / 1$ & & & & & & $4.6 \mathrm{mEq} / 1$ \\
\hline VSG & & $1-5 \mathrm{~mm}$ & & & & & & $10-27 \mathrm{~mm}$ \\
\hline Brucela & & & & Negativo & & & & \\
\hline HBsAg & & & & & & Negativo & & \\
\hline Proteus & & & & Negativo & & & & \\
\hline$\overline{\text { CORE }}$ & & & & & & Negativo & & \\
\hline$\overline{\text { HIV }}$ & & & & & & Negativo & & \\
\hline Somáticas & 13/12/8 & $\begin{array}{l}\text { O.C: Nega } \\
\text { O.A: Nega } \\
\text { O.B: Nega } \\
\text { O.D: Nega }\end{array}$ & & & & & & \\
\hline Flagelares & $13 / 12 / 8$ & $\begin{array}{l}9 \text { H.C: Negat } \\
\text { H.A: Nega } \\
\text { H.B: Nega } \\
\text { H.D: Nega }\end{array}$ & & & & & & \\
\hline Orina & $\begin{array}{l}\text { Leu: } 3-4 \\
\text { Se obser }\end{array}$ & $\begin{array}{l}\text { x campo } \\
\text { van cristales }\end{array}$ & de urato An & norfo & & & & \\
\hline (Obtenido d & gistro Mé & dico) & & & & & & \\
\hline
\end{tabular}


Tabla 2

\begin{tabular}{llc}
\hline $\begin{array}{c}\text { Tasa de Seroconversión (Infección Aguda en Profesionales Expuestos). } \\
\text { Expresadas por 100 Exposiciones de Fuente Positiva Específica. Año 1996 }\end{array}$ \\
\hline Tasa de Seroconversión para VIH-1 & 0 & $(0 / 221)$ \\
Tasa de Seroconversión para VHB & 0 & $(0 / 111)$ \\
Tasa de Seroconversión para VHC & 0,21 & $(1 / 461)$ \\
(Obtenido en: $3 \cdot^{a}$ edición, 1997. GERABTAS.) & & \\
\hline
\end{tabular}

\section{DISCUSIÓN Y CONCLUSIONES}

El personal de salud (PS) sufre alrededor de 2 millones de pinchazos con agujas (PA) anualmente, que resultan en infecciones por hepatitis B y C, y VIH. La OMS estima que la carga global de las enfermedades por exposición ocupacional entre el PS corresponde en un $40 \%$ a las infecciones por hepatitis B y C y un $2.5 \%$ de las infecciones por VIH.

Por categoría, el personal de enfermería se encuentra más expuesto a accidentes laborales por pinchazos durante su jornada de trabajo, el caso expuesto confirma las teorías anteriores. Los riesgos de transmisión de infecciones de una persona infectada a un trabajador luego de un PA son: Hepatitis B con 3-10\%, Hepatitis C de 3\% y HIV hasta un $0.3 \%$.

Para poder comprender este caso en profundidad, se debe ubicar el hipotético lector en los años donde ocurren los hechos, donde no se tenía al alcance la posibilidad de diagnosticar la Hepatitis C, si no como Hepatitis no A/ no B. Un diagnóstico por exclusión, usado para aquellas hepatitis que cursaban como tal pero que a la luz de los conocimientos de esa fecha, no se podía identificar. Fue en la década de los años 80, cuando los investigadores de los Centros para el Control de las Enfermedades (dirigidos por Daniel W. Bradley) y de Chiron (Michael Houghton) identificaron el virus. "En 1990, los bancos de sangre comenzaron a analizar la sangre de los donantes para detectar la presencia del virus. Ese mismo año, los bancos de sangre comenzaron a analizar la sangre de los donantes para detectar la presencia del VHC, pero no fue hasta 1992 cuando se perfeccionó una prueba de sangre que filtraba con eficacia el suministro de sangre destinado a transfusiones» ${ }^{3}$. Por ello en el momento del accidente laboral se realizaron las pruebas de screening a paciente fuente y a trabajadora afectada sin encontrarse ningún virus específico, solo alteraciones hepáticas que fueron normalizando con el tratamiento médico.

Entre 1989 hasta 1996 no se hace un seguimiento exhaustivo de la paciente. Esto podría fundamentarse en la escasa capacidad de localización en aquellas fechas, donde pocas personas contaban con teléfono en casa, mucho menos se podían manejar registros electrónicos de ubicación, dirección, etc. De forma no muy eficaz se contaba con la información obtenida en los registros clínicos y en ayuntamientos.

Hasta 1994, no se instaura en el hospital de Ceuta, un servicio encargado de la prevención y control de enfermedades tanto de sus propios trabajadores como del manejo de enfermedades infecciosas de usuarios de los servicios de salud, por lo que no se contaba con un protocolo de actuación eficaz en estos casos. La Dirección General de Planificación Sanitaria del Ministerio de Sanidad y Consumo aprueba su cartera de servicios y a través del RD 1277/2003, establece las bases genéricas sobre autorización de centros, servicios y establecimientos sanitarios, permitiendo la aplicación de protocolos de estudio y seguimiento, cuestión que nos permite entender la evolución de la Medicina Preventiva, Salud Pública y la Medicina del Trabajo, destacando su importancia en el contexto de las enfermedades infecciosas y su correcto control y seguimiento ya que entre sus funciones contempla el análisis epidemiológico de los datos a efectos de identificar sus causas, factores de riesgo, la tendencia y sus cambios, además de la 
notificación a las autoridades competentes del resultado de la investigación epidemiológica y las medidas de control establecidas ${ }^{4}$. Lo que podría justificar la declaración como enfermedad profesional en el año 2004 el evento descrito por la trabajadora, una vez comprobada la relación entre el paciente fuente y la trabajadora afectada.

El análisis de este caso nos permite evaluar los avances de la medicina en sus diferentes ramas a través del tiempo, orientados a la resolución de problemas y a la necesidad de implantar protocolos de actuación frente a las situaciones que se plantean en el trabajo diario, garantizando una atención mejorada de los trabajadores y su defensa frente a este tipo de incidentes. Así mismo permite determinar la influencia de los avances científicos en la mejora de calidad en atención al paciente y a los trabajadores en general.

La definición legal de enfermedad profesional, actualmente recogida en el art. 116 de la Ley General de los Seguros Sociales (LGSS) de 1994, conforme a la cual se considera tal: la contraída a consecuencia del trabajo ejecutado por cuenta ajena en las actividades que se especifiquen en el cuadro que se apruebe por las disposiciones de aplicación y desarrollo de esta ley, y que esté provocada por la acción de los elementos o sustancias que en dicho cuadro se indiquen para cada enfermedad profesional's.

Por el cumplimiento de estos criterios no se declara como enfermedad profesional la Hepatitis C contraída con motivo del pinchazo, hasta comprobarse la infección del paciente fuente con el mismo genotipo (1b) en el año 2004.

Las alteraciones de la salud debidas a Agentes biológicos son tan diversas, complejas y variables que dificultan un enfoque exhaustivo y hacen necesaria una herramienta de trabajo para realizar la vigilancia de salud que conjugue generalidad e individualidad, razón que justifica la aplicación de un protocolo de riesgos biológicos, los cuales recogen la forma de actuación del encargado de la vigilancia sanitaria en caso de exposición accidental, todo ellos basados en los datos obtenidos en diversos estudios tales como: GERABTAS (Grupo Español de Registro de Accidentes Biológicos en Trabajadores de Atención de Salud), EPINETAC (Exposure Prevention Information Network), entre otros, que surgen por la necesidad de establecer de forma científica el grado de exposición, el número de seroconversiones y los agentes biológicos a los que en mayor medida están expuestos los trabajadores, con esta base crear mecanismos de protección y vigilancia, instaurando medidas más eficaces en la prevención de accidentes y por ende de las enfermedades derivadas.

El GERABTAS, fue un estudio prospectivo de los accidentes ocurridos en 1994 en 70 centros asistenciales de toda España que observó una tasa de exposición laboral (expresada por 1000 personas-año) de 51 para el conjunto de los trabajadores sanitarios, correspondiendo los valores más elevados a las enfermeras (91). El 14.4\% de las exposiciones fueron a fuente positiva; de ellas $3,1 \%$ correspondieron a $\mathrm{VHB}, 5,8 \%$ al $\mathrm{VIH}$ y $11,5 \%$ al VHC.

También se pudo constatar en España una incidencia de seroconversión de 0,33\% (2 seroconversiones en 603 expuestos a sangre positiva al VHC. Cada vez sin embargo, tiene más importancia la hepatitis $\mathrm{C}$, por un lado por su gravedad, dada la tendencia a evolucionar a formas crónicas, cirrosis y hepatocarcinoma y por otro porque la tasa de exposición a fuente positiva a VHC (7,8/1000 personas-año) es mayor que las correspondientes al VHB $(2,1)$ y al VIH $(3,9 \%)$ lo que se refleja en la frecuencia de enfermos VHC positivos ingresados en los hospitales ${ }^{6}$.

Así mismo el GERABTAS al estudiar las tasas de seroconversión (infección aguda en profesionales expuestos), en el análisis con periodo de seguimiento para investigar seroconversiones en personal sanitario, solamente ha registrado 1 seroconversión al VHC en toda la serie, no existiendo hasta el momento del estudio ninguna seroconversión al VHB, ni para VIH1 para un total de 3417 protocolos cerrados ${ }^{7}$. (Ver tabla 2). Dicho caso coincide con el EPINETAC-1997, donde se realiza una descripción detallada del mismo $^{8}$. 
Según real decreto 664/1997, de 12 de mayo, sobre la protección de los trabajadores contra los riesgos relacionados con la exposición a agentes biológicos durante el trabajo. En cuanto al manejo de la documentación, es importante destacar la obligatoriedad de conservar la lista de los trabajadores expuestos y los historiales médicos durante un plazo mínimo de 10 años después de finalizada la exposición; este plazo se ampliará hasta cuarenta años en caso de exposición que pudieran dar lugar a una infección que no sea diagnosticable con los conocimientos actuales, hasta la manifestación de la enfermedad muchos años después. Basados en la evolución cronológica del caso expuesto, se manifiesta la necesidad de este tipo de legislaciones, ya que a la trabajadora se le pudo diagnosticar su enfermedad profesional 15 años después, cuando los adelantos científicos pudieron determinar la enfermedad y su relación con el paciente?

El Accidente de Trabajo, desde la perspectiva legal, queda definido en el artículo 115 del Texto Refundido de la Ley General de la Seguridad Social (RD Legislativo 1/1994, de 20 de junio), de la siguiente forma: Se entiende por accidente de trabajo toda lesión corporal que el trabajador sufra como consecuencia del trabajo que ejecute por cuenta ajena ${ }^{10}$.

Los riesgos se pueden relacionar con las características del instrumento, pero la mayor parte de las lesiones por pinchazos están relacionadas con malas praxis de trabajo como: volver a encapuchar las agujas, transferir de un recipiente a otro un fluido corporal (transferir sangre de una jeringa a un tubo) y no eliminar los instrumentos cortopunzantes en un recipiente adecuado. Tener en consideración las medidas de protección frente a pinchazos en el medio hospitalario para evitar este tipo de accidentes es fundamental, ya que a pesar de los avances y las medidas de prevención, los pinchazos accidentales siguen siendo un tema importante de la enfermería a nivel mundial, con consecuencias graves en la salud de los trabajadores y costos muy elevados para el sistema sanitario.

Valorar la evolución cronológica de los avances científicos en la medicina y el análisis de datos estadísticos, nos permite conocer la importancia de las revisiones de los hechos cotidianos del campo laboral con rigor científico ya que en base a dichos resultados se deciden las diferentes estrategias para mejorar la vigilancia y control de las enfermedades laborales, incidiendo directamente en la calidad de vida de los trabajadores y en el reconocimiento de sus derechos.

\section{REFERENCIAS BIBLIOGRÁFICAS}

1. Beekmann SE, Henderson DK. Protection of healthcare workers from blood borne pathogens. Curr Opin Infect Dis 2005;18: 331-336.

2. Wilburn S. La Prevención de Pinchazos con Agujas en el Personal de Salud. Disponible en: http://www. who.int/occupational_health/activities/oehcdrom5.pdf.

3. Franciscus A. Breve Historia de la Hepatitis C. Disponible en: http://www.hcvadvocate.org/pdf/historia. pdf.

4. Ministerio de Sanidad y Consumo. Real Decreto $1277 / 2003$, de 10 de octubre, por el que se establecen las bases generales sobre autorización de centros, servicios y establecimientos sanitarios. Disponible en: http://www.boe.es/boe/dias/2003/10/23/pdfs/A37893-37902.pdf.

5. Caras Martínez F. Las enfermedades profesionales desde la perspectiva de la seguridad social. Disponible en: http://www.seg-social.es/prdi00/groups/public/documents/binario/115799.pdf.

6. Benavides, F. Salud Laboral. Conceptos y Técnicas para la Prevención de Riesgos Laborales. Barcelona: Masson, S.A; 1997.

7. Monge Jodra, V. Accidentes Biológicos en Profesionales Sanitarios. GERABTAS 3ra ed. Madrid; 1997.

8. Arribas Llorente, J. Estudio y Seguimiento del Riesgo Biológico en el Personal Sanitario. Proyecto EPINETAC 1998-2000. Grupo de Trabajo EPINETAC. Capítulo 4.

9. Instituto Navarro de Salud Laboral. Protección de los Trabajadores contra los riesgos relacionados con la exposición a Agentes Biológicos durante el trabajo. Marzo 2011.

10. Disponible en: http://www.navarra.es/NR/rdonlyres/8B31D913-389D-4244-87C9-E395F865135F/185019/ Agentes. 
11. Ministerio de Empleo y Seguridad Social. Real Decreto Legislativo 1/1994, de 20 de junio, por el que se aprueba el Texto Refundido de la Ley General de la Seguridad Social. Disponible en: http://www.segsocial.es/Internet_1/Normativa/095093?ssSourceNodeId=1139\#A115.

12. Gestal Otero, J. Riesgos del Trabajo del Personal Sanitario. Madrid: Interamericana McGraw Hill; 1993. 